\title{
SEMI-BLIND CHANNEL IDENTIFICATION AND SYMBOL ESTIMATION FOR ASYNCHRONOUS MIMO SYSTEMS
}

Yonghong Zeng, Member, IEEE

\author{
Institute for Infocomm Research, A-STAR \\ 21 Heng Mui Keng Terrace \\ Singapore 119613 \\ Email: yhzeng@ieee.org
}

\begin{abstract}
SEMI-BLIND channel identification and symbol estimation for asynchronous MIMO systems are considered in this paper. MIMO channels are estimated from the second order statistics of the received signals subject to an ambiguity matrix and unknown time delays. Then a small number of pilot symbols are used to resolve the ambiguity matrix and time delays. A two-step symbol estimation method, which estimates the channels before recovering the symbols, is proposed. Only upper bounds for the channel orders and time delays are needed for implementing the algorithms. Neither knowledge of real channel orders nor precise synchronization of different users is required, which makes the algorithm applicable to practical MIMO systems.
\end{abstract}

\section{INTRODUCTION}

Multiple input multiple output (MIMO) system has the potential to greatly increase system capacity and therefore is considered as a major technique for future wireless communication systems. Since there are multiple users and signals propagate through different channels usually have different time delays, it is virtually impossible to precisely synchronize an MIMO system. The situation is even more obvious in the uplink of a cellular mobile system. Therefore, a MIMO system is usually an asynchronous system. Although quite a few methods have been proposed for MIMO channel estimation and equalization $[1,6,7,9]$, most of them have two restrictions. One is the requirement of the first coefficient (a matrix) in the $\mathrm{Z}$ transform representation of the MIMO matrix channel to be of full column rank which implicitly implies precise synchronization [3]. The other is the true channel orders are known $[4,5]$. In [9], a blind channel estimation method for synchronous MIMO systems is proposed which only required an upper bound of the channel orders. For a MIMO system, it is virtually impossible to achieve precise synchronization and obtain the exact channel orders. Very few work has been published on equalizing asynchronous MIMO systems with multipath channels.

This work is supported by a grant, HKU 7164/04E, from the Research Grants Council of the Hong Kong SAR, China.

\author{
Shaodan Ma and Tung Sang Ng, Fellow,IEEE
}

\author{
Department of Electrical and Electronic Engineering \\ The University of Hong Kong \\ Pokfulam Road, Hong Kong \\ Email: sdma@eee.hku.hk,tsng@eee.hku.hk
}

In this paper, an asynchronous MIMO system with multipath channels is considered, which allows different channels to have different time delays. By shifting the channels, we turn the system into a form in which only few time delays need to be estimated. Channels and transmitted symbols of the transformed system can be blindly estimated by some known methods with an ambiguity matrix and the time delays embedded. For estimating the time delays and the ambiguity matrix, pilot symbols are necessary and a method is proposed. Major features of the methods are: (1) precise synchronization of different channels is not required; (2) only an upper bound for the multipath channel orders is needed for implementation; (3) time delays for the multiple channels can be different and unknown, and only an upper bound for all the time delays is required. These features make the algorithm applicable to practical MIMO systems. Simulations show that the algorithms are effective and robust.

The rest of the paper is organized as follows. In Section 2, the asynchronous MIMO system model and its transformation are discussed. The channel identification algorithm is presented in Section 3. Section 4 proposes a semi-blind two-step symbol estimation method. Some simulation results are given in Section 5. Finally, conclusions are drawn in Section 6.

In the following, superscripts $T, \dagger$ and $*$ stand for transpose, Hermitian (transconjugate), and conjugate, respectively. Symbol $\stackrel{\text { def }}{=}$ is used for introducing a new notation. $\mathbf{I}_{q}$ is the identity matrix of order $q$ and $\otimes$ is the Kronecker product of matrices.

\section{SYSTEM MODEL}

Consider an asynchronous MIMO system with $P$ users and $M$ receivers (antennas). Each user sends a symbol sequence: $s_{j}(n)(j=1,2, \cdots, P)$. Assume that the transmitted symbols have zero expectations and are independently and identically distributed. In general, let the time delay of the channel from user $j$ to antenna $i$ be $d_{i j}$. Then the received signal in the $i$ th receiver (antenna) can be de- 
scribed as

$$
\begin{aligned}
x_{i}(n) & =\sum_{j=1}^{P} \sum_{k=0}^{N_{i j}} h_{i j}(k) s_{j}\left(n-k-d_{i j}\right)+\eta_{i}(n), \\
& =\sum_{j=1}^{P} \sum_{k} h_{i j}\left(k-d_{i j}\right) s_{j}(n-k)+\eta_{i}(n),(1)
\end{aligned}
$$

where $h_{i j}(k)$ is the channel response from user $j$ to antenna $i, N_{i j}$ is the order of channel $h_{i j}(k)\left(h_{i j}(0) \neq 0\right.$, $h_{i j}\left(N_{i j}\right) \neq 0$ ), and $\eta_{i}(n)$ is the channel noise, which is white and uncorrelated with the transmitted signals. Obviously, the actual (effective) channel responses are $h_{i j}(k-$ $\left.d_{i j}\right)$ - thus the first coefficient of the MIMO matrix channel is matrix $\left[h_{i j}\left(-d_{i j}\right)\right]_{i j}$ and it is not of full column rank in most cases. Therefore, most known methods $[1,6,7,9]$ cannot be used for the system (see [3] for more explanations). In the following, we will transform the system into another form which enables some of the known methods to be applicable.

Defining $h_{i j}(k)=0$ for $k<0$ or $k>N_{i j}$ and $d_{j} \stackrel{\text { def }}{=}$ $\min _{i}\left(d_{i j}\right)$,we have

$$
x_{i}(n)=\sum_{j=1}^{P} \sum_{k} \hat{h}_{i j}(k) \hat{s}_{j}(n-k)+\eta_{i}(n),
$$

where

$$
\hat{h}_{i j}(k)=h_{i j}\left(k-d_{i j}+d_{j}\right), \hat{s}_{j}(n)=s_{j}\left(n-d_{j}\right) .
$$

Let $\hat{N}_{j} \stackrel{\text { def }}{=} \max _{i}\left(N_{i j}+d_{i j}\right)-d_{j}$. Then it is obvious that $\hat{h}_{i j}(k)=0$ if $k<0$ or $k>\hat{N}_{j}$. Hence (2) can be written as

$$
x_{i}(n)=\sum_{j=1}^{P} \sum_{k=0}^{\hat{N}_{j}} \hat{h}_{i j}(k) \hat{s}_{j}(n-k)+\eta_{i}(n) .
$$

We assume that $M>P$. Letting

$$
\begin{aligned}
& \mathbf{x}(n) \stackrel{\text { def }}{=}\left[x_{1}(n), x_{2}(n), \cdots, x_{M}(n)\right]^{T}, \\
& \hat{\mathbf{h}}_{j}(n) \stackrel{\text { def }}{=}\left[\hat{h}_{1 j}(n), \hat{h}_{2 j}(n), \cdots, \hat{h}_{M j}(n)\right]^{T}, \\
& \eta(n) \stackrel{\text { def }}{=}\left[\eta_{1}(n), \eta_{2}(n), \cdots, \eta_{M}(n)\right]^{T},
\end{aligned}
$$

we can express (4) into vector form as

$$
\mathbf{x}(n)=\sum_{j=1}^{P} \sum_{k=0}^{\hat{N}_{j}} \hat{\mathbf{h}}_{j}(k) \hat{s}_{j}(n-k)+\eta(n), n=0,1, \cdots .
$$

Considering $L$ consecutive outputs and defining

$$
\begin{aligned}
\hat{\mathbf{x}}(n) \stackrel{\text { def }}{=} & {\left[\mathbf{x}^{T}(n), \mathbf{x}^{T}(n-1), \cdots, \mathbf{x}^{T}(n-L+1)\right]^{T}, } \\
\hat{\eta}(n) \stackrel{\text { def }}{=} & {\left[\eta^{T}(n), \eta^{T}(n-1), \cdots, \eta^{T}(n-L+1)\right]^{T}, } \\
\hat{\mathbf{s}}(n) \stackrel{\text { def }}{=} & {\left[\hat{s}_{1}(n), \cdots, \hat{s}_{1}\left(n-N_{1}-L+1\right), \cdots,\right.} \\
& \\
& \left.\hat{s}_{P}(n), \cdots, \hat{s}_{P}\left(n-N_{P}-L+1\right)\right]^{T},
\end{aligned}
$$

we get

$$
\hat{\mathbf{x}}(n)=\hat{\mathbf{H}} \hat{\mathbf{s}}(n)+\hat{\eta}(n),
$$

where $\hat{\mathbf{H}}$ is an $M L \times(\hat{N}+P L)\left(\hat{N} \stackrel{\text { def }}{=} \sum_{j=1}^{P} \hat{N}_{j}\right)$ matrix defined as

$$
\begin{aligned}
& \hat{\mathbf{H}} \stackrel{\text { def }}{=}\left[\hat{\mathbf{H}}_{1}, \hat{\mathbf{H}}_{2}, \cdots, \hat{\mathbf{H}}_{P}\right], \\
& \hat{\mathbf{H}}_{j} \stackrel{\text { def }}{=}\left[\begin{array}{cccccc}
\hat{\mathbf{h}}_{j}(0) & \ldots & \hat{\mathbf{h}}_{j}\left(\hat{N}_{j}\right) & 0 & \cdots & 0 \\
0 & \hat{\mathbf{h}}_{j}(0) & \cdots & \hat{\mathbf{h}}_{j}\left(\hat{N}_{j}\right) & \cdots & 0 \\
& & \ddots & & \ddots & \\
0 & 0 & \cdots & \hat{\mathbf{h}}_{j}(0) & \cdots & \hat{\mathbf{h}}_{j}\left(\hat{N}_{j}\right)
\end{array}\right]
\end{aligned}
$$

\section{BLIND CHANNEL IDENTIFICATION}

The system model (8) is similar to that in [9] with $\mathbf{H}$ and $\mathbf{s}$ replaced by $\hat{\mathbf{H}}$ and $\hat{\mathbf{s}}$, respectively. To use the results in [9], the matrix $\hat{\mathbf{H}}$ must be of full column rank. If $M>P$ and the smoothing factor $L>\hat{N} /(M-P)$, the matrix has more rows than columns. Therefore, it is most likely of full column rank and some results in [9] can be readily used. Using the method in [9], we can derive a method for estimating the $\hat{h}_{i j}(k)$, which is a delayed version of $h_{i j}(k)$. If the estimated channels are used for equalization, obtaining $\hat{h}_{i j}(k)$ is enough and there is no need to know $h_{i j}(k)$. However, to finally obtain the symbols $s_{j}(n)$, the delay $d_{j}$ (not all $d_{i j}$ ), the minimum delay of the $M$ channels for user $j$, must be found. The resolving of the $d_{j}$ will be discussed in the next section. The algorithm for estimating the $\hat{h}_{i j}(k)$ is given in the following. Note that it is an improved version of [9] by incorporating the maximum description length (MDL) method [8].

\section{Algorithm 1 : Blind channel identification for asyn- chronous MIMO systems}

It is assumed that an upper bound for all the channel orders, that is, a number $N_{\text {upp }}$ such that $\hat{N}_{j} \leqslant N_{u p p}(j=$ $1,2, \cdots, P)$, is known or estimated. Choose a smoothing factor $L>P N_{\text {upp }} /(M-P)$.

Step 1. Compute $\mathbf{R}=\frac{1}{L_{s}} \sum_{n=L}^{L+L_{s}-1} \hat{\mathbf{x}}(n) \hat{\mathbf{x}}^{\dagger}(n)$, where $L_{s}$ is the number of samples used. Compute the eigenvalue decomposition (EVD) of $\mathbf{R}$. Use the MDL method [8] to estimate the rank of $\mathbf{H}$. Let the estimated rank be $r$. Average the smallest $M L-r$ eigenvalues of $\mathbf{R}$ to get an estimation $\left(\bar{\sigma}_{\eta}^{2}\right)$ for the noise variance. Let $\overline{\mathbf{R}}=$ $\mathbf{R}-\bar{\sigma}_{\eta}^{2} \mathbf{I}_{M L}$.

Step 2. Compute $\mathbf{Q}=\frac{1}{L_{s}} \sum_{n=L}^{L+L_{s}-1} \hat{\mathbf{x}}(n) \hat{\mathbf{x}}^{\dagger}(n-1)$ and $\mathbf{Q}=\mathbf{Q}-\bar{\sigma}_{\eta}^{2}\left(\mathbf{J}_{L} \otimes \mathbf{I}_{M}\right)$. Then compute the singular value decomposition (SVD) of $\overline{\mathbf{Q}}$. Let $K_{1}=M L-r+$ $P$. Choose $K_{1}$ singular vectors $\mathbf{u}_{i}\left(i=1,2, \cdots, K_{1}\right)$ corresponding to the $K_{1}$ least left singular values of $\overline{\mathbf{Q}}$ and denote a matrix $\mathbf{U}=\left[\mathbf{u}_{1}, \mathbf{u}_{2}, \cdots, \mathbf{u}_{K_{1}}\right]$.

Step 3. Compute $\mathbf{W}=\mathbf{U}^{\dagger} \overline{\mathbf{R}} \mathbf{U}$ and the $E V D$ of $\mathbf{W}$. Let $\mathbf{V}_{1}$ be the matrix of size $K_{1} \times P$ whose columns are eigenvectors corresponding to nonzero eigenvalues of $\mathbf{W}$, and $\mathbf{U}_{1}=\mathbf{U V}_{1}$.

Step 4. For $k=0,1, \cdots, N_{\text {upp }}+L-1$, compute

$$
\mathbf{r}_{z}(k)=\left\{\begin{array}{ll}
\mathbf{r}_{x}(k), & k \neq 0 \\
\mathbf{r}_{x}(k)-\bar{\sigma}_{\eta}^{2} \mathbf{I}_{M}, & k=0
\end{array},\right.
$$


where $\mathbf{r}_{x}(k)=\frac{1}{L_{s}} \sum_{n=k}^{k+L_{s}-1} \mathbf{x}(n) \mathbf{x}^{\dagger}(n-k)$.

Step 5. For $k=0,1, \cdots, N_{\text {upp }}$, let $\mathbf{G}(k)=\left[\begin{array}{llll}\mathbf{r}_{z}(k) & \mathbf{r}_{z}(k+1) & \cdots & \mathbf{r}_{z}(k+L-1)\end{array}\right] \mathbf{U}_{1}$.

The MIMO channel matrix is then $\hat{\mathbf{h}}(k)=\mathbf{G}(k) \mathbf{B}^{-1}$, where $\mathbf{B}$ is a $P \times P$ matrix to be determined, and

$$
\hat{\mathbf{h}}(k)=\left[\begin{array}{ccc}
\hat{h}_{11}(k) & \cdots & \hat{h}_{1 P}(k) \\
\vdots & \cdots & \vdots \\
\hat{h}_{M 1}(k) & \cdots & \hat{h}_{M P}(k)
\end{array}\right] .
$$

\section{TWO-STEP SYMBOL ESTIMATION}

There are an ambiguity matrix $\mathbf{B}$ (in the estimated channels) and delays $d_{j}$ to be resolved. For resolving them, pilot symbols are necessary. In this section, a pilot-based method is proposed and then a two-step symbol estimation method is discussed.

\subsection{Estimation of the time delays}

Let

$$
\overline{\mathbf{s}}(n)=\left[s_{1}\left(n-d_{1}\right) s_{2}\left(n-d_{2}\right) \cdots s_{P}\left(n-d_{P}\right)\right]^{T} .
$$

A linear minimum mean square error (MMSE) equalization can be constructed as

$$
\overline{\mathbf{s}}(n-\gamma)=\overline{\mathbb{H}}_{\gamma}^{\dagger} \mathbf{R}^{-1} \hat{\mathbf{x}}(n),
$$

where $\overline{\mathbb{H}}_{\gamma}$ is a matrix of size $M L \times P$ which is constructed from the channel responses $\hat{\mathbf{h}}(k)$ and $\gamma$ is a delay, $0 \leqslant$ $\gamma \leqslant N_{\text {upp }}+L-1$ (see [2], page 341). Let $\overline{\mathbb{G}}_{\gamma}$ be defined similarly as $\overline{\mathbb{H}}_{\gamma}$ from $\mathbf{G}(k)$. From Algorithm 1 (Step 5), it is obvious that $\overline{\mathbb{H}}_{\gamma}=\overline{\mathbb{G}}_{\gamma} \mathbf{B}^{-1}$. Therefore,

$$
\mathbf{B}^{\dagger} \overline{\mathbf{s}}(n-\gamma)=\overline{\mathbb{G}}_{\gamma}^{\dagger} \mathbf{R}^{-1} \hat{\mathbf{x}}(n),
$$

that is,

$$
\mathbf{B}^{\dagger} \overline{\mathbf{s}}(n)=\mathbf{y}(n),
$$

where $\mathbf{y}(n)=\overline{\mathbb{G}}_{\gamma}^{\dagger} \mathbf{R}^{-1} \hat{\mathbf{x}}(n+\gamma)$. Please note that $\mathbf{y}(n)$ can be computed from the outputs and estimated channels $\mathbf{G}(k)$.

Let

$$
\mathbf{T}(k) \stackrel{\text { def }}{=} \mathrm{E}\left(\mathbf{y}(n) \mathbf{s}^{\dagger}(n-k)\right),
$$

where $\mathrm{E}(\xi)$ means the mathematical expectation of a random variable $\xi$ and

$$
\mathbf{s}(n)=\left[s_{1}(n) s_{2}(n) \cdots s_{P}(n)\right]^{T} .
$$

From (14) we have

$$
\mathbf{T}(k)=\mathbf{B}^{\dagger} \mathrm{E}\left(\overline{\mathbf{s}}(n) \mathbf{s}^{\dagger}(n-k)\right) .
$$

It is clear that

$\mathrm{E}\left(\overline{\mathbf{s}}(n) \mathbf{s}^{\dagger}(n-k)\right)= \begin{cases}0, & k \neq d_{j}(j=1,2, \cdots, P) \\ \boldsymbol{\Delta}_{j}, & k=d_{j}\end{cases}$ where $\boldsymbol{\Delta}_{j}$ is a matrix with all elements being zeros except that the element at $j$-th row and $j$-th column being 1. Therefore, if $k \neq d_{j}(j=1,2, \cdots, P), \mathbf{T}(k)$ must be zero. If $k=d_{j}$, the $j$-th column of $\mathbf{T}(k)$ is the $j$-th column of $\mathbf{B}^{\dagger}$ and all the other columns are zeros. Since $\mathbf{B}^{\dagger}$ is invertible, none of its column is zero. Let $m$ be a number such that $m \geq d_{j}(j=1,2, \cdots, P)$, which can be obtained from some knowledge on the time delays. If we can obtain $\mathbf{T}(k)(k=0,1, \cdots, m)$, the delays $d_{j}$ can be easily found $\left(d_{j}\right.$ is the only $k$ such that the $j$-th column of $\mathbf{T}(k)$ is nonzero). In practice, $\mathbf{T}(k)$ can only be estimated (with errors) from a finite number of pilot samples, and therefore we should choose $d_{j}$ to be the $k$ such that the power of the $j$-th column of $\mathbf{T}(k)$ is maximized.

\subsection{Symbol estimation}

To use (12) for symbol estimation, the ambiguity matrix $\mathbf{B}$ in the estimated channels needs to be resolved. The same pilots for resolving the time delays can be used here. In fact, defining

$$
\begin{aligned}
& \mathbf{S}=[\overline{\mathbf{s}}(m) \overline{\mathbf{s}}(m+1) \cdots \overline{\mathbf{s}}(m+J-1)] \\
& \mathbf{Y}=[\mathbf{y}(m) \mathbf{y}(m+1) \cdots \mathbf{y}(m+J-1)]
\end{aligned}
$$

From (14) we have

$$
\mathbf{B}^{\dagger} \mathbf{S}=\mathbf{Y}
$$

A least square (LS) estimation is then

$$
\mathbf{B}^{\dagger}=\mathbf{Y} \mathbf{S}^{\dagger}\left(\mathbf{S S}^{\dagger}\right)^{-1} .
$$

With the estimated channel responses $\hat{\mathbf{h}}(k)$, the ambiguity matrix $\mathbf{B}$ and the time delays, the transmitted signals can be recovered using (12). In this two-step method, $m+J$ pilot symbols are needed for each user.

\section{SIMULATIONS}

Consider a 2-user 4-antenna system $(M=4, P=2)$. The channel orders are 3 (for user 1) and 4 (for user 2) respectively (note that the two users have different channel orders). The channel responses (without the time delays $d_{i j}$ ) $h_{i j}(k)$ are generated randomly. For each user, the modulation scheme is 4-QAM. The time delays are: $d_{11}=$ $d_{21}=d_{31}=d_{41}=0, d_{12}=d_{22}=d_{42}=1, d_{32}=2$. Hence $\hat{N}_{1}=3, \hat{N}_{2}=5, N_{\max } \stackrel{\text { def }}{=} \max _{j} \hat{N}_{j}=5$ and $d_{1}=0, d_{2}=1$. Only an upper bound for all the orders is assumed known in our simulations, that is, a number $N_{u p p}$ is known such that $N_{u p p} \geq N_{\max }$. To verify the robustness of the algorithm to the channel order overestimation, we test four cases of the channel order upper bound $\left(N_{\text {upp }}\right): N_{\max }$ (exact upper bound), $N_{\max }+2, N_{\max }+4$ and $N_{\max }+6$, respectively. The signal-noise-ratio (SNR) as the ratio of the average received signal power to the average noise power is defined as

$$
\mathrm{SNR} \stackrel{\text { def }}{=} \frac{\mathrm{E}\left(\|\mathbf{x}(n)-\eta(n)\|^{2}\right)}{\mathrm{E}\left(\|\eta(n)\|^{2}\right)} .
$$


The MSE between the estimated and true channel responses is defined as

$$
\operatorname{MSE} \stackrel{\text { def }}{=} \min _{\beta} \frac{\sum_{l=0}^{N_{u p p}}\|\hat{\mathbf{h}}(l)-\mathbf{G}(l) \beta\|_{F}^{2}}{\sum_{l=0}^{N_{u p p}}\|\hat{\mathbf{h}}(l)\|_{F}^{2}} .
$$

Simulations show that the algorithm is truly robust to channel order overestimation, noise and round-off errors.

The MSE versus SNR is shown in Figure 1. We see that the algorithm works well when only an upper bound for all the channel orders is known. When the channel orders are overestimated, errors are inevitably introduced to the channel tails (ideally should be zeros), which causes the MSE to become higher. Figure 2 shows the bit error

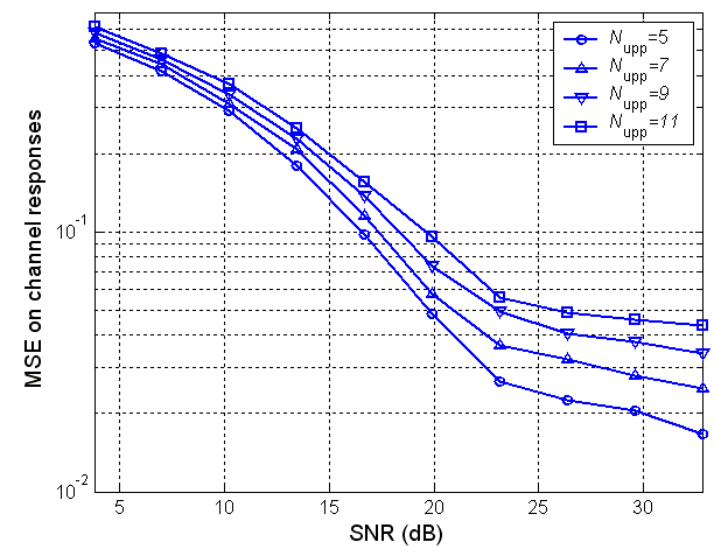

Fig. 1. MSE versus $\operatorname{SNR}\left(L_{s}=500\right)$

rate (BER) (average of all users) versus SNR when the two-step method is used. An upper bound for time delays is chosen as $m=\max _{j}\left(d_{j}\right)+3$, that is, the time delays are overestimated by at least 3. The delay $\gamma$ is chosen to be $N_{\text {upp }}$, and for comparison, the BER results using the true channels and delays are also given (see the line with no mark). It is clear that the symbol estimation algorithm works well when only upper bounds for the channel orders and time delays are known. Furthermore, the assumed upper bounds can be much larger than the exact upper bounds.

\section{CONCLUSIONS}

In this paper, semi-blind channel identification and symbol estimation algorithms for asynchronous MIMO systems have been proposed. MIMO channels and transmitted symbols are first estimated from the SOS of the received signals subject to an ambiguity matrix and unknown time delays. Some pilot symbols are then used to resolve the ambiguity matrix and the time delays. The algorithm requires neither knowledge of real channel orders nor precise synchronization of different users, which makes the algorithm practical for applications. Simulations have shown that the algorithms are effective and robust.

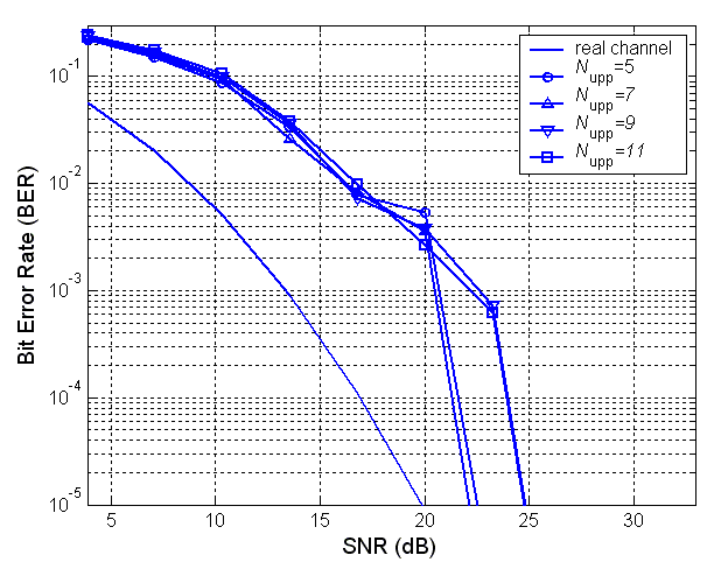

Fig. 2. BER versus SNR (two-step method, $L_{s}=1000$ )

\section{REFERENCES}

[1] I. Bradaric, A. P. Petropulu and K. I. Diamantaras, On blind identifiability of FIR-MIMO systems with cyclostationary inputs using second order statistics, IEEE Trans. Signal Processing, vol. 51, no. 2, pp. 434-441, 2003.

[2] Z. Ding and Y. Li, Blind Equalization and Identification, Marcel Dekker, Inc., New York, 2001.

[3] Y. Inouye and R. W. Liu, A system-theoretic foundation for blind equalization of an FIR MIMO channel system, IEEE Trans. Circuits Systems -I, vol.49, pp.425-436, Apr. 2002

[4] A. P. Liavas, P. A. Regalia, and J. P. Delmas, On the robustness of the linear prediction method for blind channel identification with respect to effective channel undermodeling/overmodeling, IEEE Trans. Signal Processing, vol. 48, pp. 1477-1481, May 2000.

[5] W. Qiu and Y. Hua, Performance analysis of the subspace method for blind channel identification, Signal Processing, vol. 50, pp. 71-81, 1996.

[6] N. Thirion-Moreau and E. Moreau, Generalized criteria for blind multivariate signal equalization, IEEE Signal Processing Letters, vol.9, pp.72-74, Feb. 2002

[7] J. K. Tugnait and B. Huang, Multistep linear predictors-based blind identification and equalization of multiple-input multiple-output channels, IEEE Trans. Signal Processing, vol. 48, pp. 26-38, Jan. 2000

[8] M. Wax and T. Kailath, Detection of signals by information theoretic criteria, IEEE Trans. Acoust., Speech, Signal Processing, vol. ASSP-33, pp. 387392, Apr. 1985.

[9] Y. H. Zeng and T. S. Ng, A Blind MIMO channel estimation method robust to order overestimation, Signal Processing, vol. 84, no. 2, pp. 435-439, 2004. 